\title{
ALDH1-positive cancer stem-like cells are enriched in nodal metastases of oropharyngeal squamous cell carcinoma independent of HPV status
}

\author{
XU QIAN $^{1,2}$, STEFFEN WAGNER $^{3}$, CHENMING MA $^{1}$, JENS P. KLUSSMANN ${ }^{3}$, \\ MICHAEL HUMMEL $^{4}$, ANDREAS M. KAUFMANN ${ }^{5}$ and ANDREAS E. ALBERS ${ }^{1}$ \\ ${ }^{1}$ Department of Otorhinolaryngology, Head and Neck Surgery, Charité-Universitätsmedizin Berlin, \\ Campus Benjamin Franklin, Berlin, Germany; ${ }^{2}$ Zhejiang Provincial Key Laboratory of Medical Genetics, \\ Department of Histology and Embryology, Wenzhou Medical College, Wenzhou, P.R. China; ${ }^{3}$ University Hospital of \\ Giessen and Marburg, Department of Otorhinolaryngology, Head and Neck Surgery, Giessen; ${ }^{4}$ Institute of Pathology, \\ Charité-Universitätsmedizin Berlin, Campus Benjamin Franklin, Berlin; ${ }^{5}$ Clinic for Gynecology, \\ Charité-Universitätsmedizin Berlin, Campus Mitte and Benjamin Franklin, Berlin, Germany
}

Received November 26, 2012; Accepted December 27, 2012

DOI: $10.3892 / o r .2013 .2340$

\begin{abstract}
Oropharyngeal squamous cell carcinoma (OSCC) is caused by high-risk (HR) human papillomavirus (HPV) or alcohol and tobacco abuse. Aldehyde dehydrogenase 1 (ALDH1) is a confirmed marker for cancer stem-like cells (CSCs) of OSCC responsible for therapy resistance, recurrence and metastasis. Associations between HR-HPV/p16, CSC frequency and clinicopathological parameters in patients with metastatic OSCC were investigated. In the present study, HPV genotypes and expression of ALDH1 and p16 was analyzed in 40 paired OSCC and metastases. A significant correlation between ALDH1 positivity with lower primary tumor differentiation grade $(\mathrm{P}=0.009)$ and higher nodal status $(\mathrm{P}=0.015)$ was noted. Compared to primary tumors, the proportion of ALDH1-expressing cells was significantly increased in metastases $(\mathrm{P}=0.012)$, while significantly fewer ALDH1-expressing cells were found in $\mathrm{HR}-\mathrm{HPV}-\mathrm{DNA}{ }^{+} / \mathrm{p}^{+} 6^{+}$primary tumors $(\mathrm{P}=0.038)$ compared to $\mathrm{HR}-\mathrm{HPV}-\mathrm{DNA} / \mathrm{p}^{-} 6^{-}$primary tumors. Metastases showed no difference. $\mathrm{ALDH}^{+} \mathrm{CSC}$ are detectable in OSCC and metastases. ALDH1 high-grade OSCC exhibits a more aggressive phenotype characterized by higher nodal classification and lower differentiation. This suggests a subpopulation contained in the ALDH1-positive OSCC cell pool able to complete the metastatic cascade and subsequently enriching in metastasis independent of tumor etiology and ALDH1 content.
\end{abstract}

Correspondence to: $\operatorname{Dr}$ Andreas E. Albers, Department of Otorhinolaryngology, Head and Neck Surgery, Charité-Universitätsmedizin Berlin, Campus Benjamin Franklin, Hindenburgdamm 30, D-12200 Berlin, Germany

E-mail: andreas.albers@charite.de

Key words: squamous cell carcinoma, human papillomavirus, cancer stem cell, aldehyde-dehydrogenase, metastasis

\section{Introduction}

Head and neck squamous cell carcinoma (HNSCC) is the sixth most common type of cancer worldwide and only $40-50 \%$ of patients survive 5 years after initial diagnosis (1). Among HNSCC, incidence of high-risk (HR)-human papillomavirus (HPV)-positive oropharyngeal squamous cell carcinoma (OSCC) has increased over the past 20 years while the incidence of HR-HPV-HNSCC has steadily decreased with reduced alcohol and tobacco consumption (1-4). The high mortality of this disease is due to the development of distant metastases and the emergence of eventually inoperable local and regional recurrences that have low responsiveness to radiation therapy or chemotherapy (1). However, if HNSCC is divided into subgroups with regard to their etiology, $\mathrm{HR}-\mathrm{HPV}^{+}$ tumors appear to be more responsive to treatment and patients show better survival rates $(5,6)$.

Identification and characterization of cancer stem cell-like cells (CSCs) in HNSCC, including OSCC, yields new insights into the possible causes of the poor prognosis. CSCs are a small subpopulation of cells within the tumor that exhibit self-renewing ability and are responsible for tumor maintenance, growth, metastasis and also for resistance towards chemotherapy and radiation therapy (7-9). Therefore, tumor cells can be divided into two subpopulations, a bulk population of non-CSCs and a smaller population of CSCs (10). CSCs have been identified by appropriate marker expressions such as CD44 (11). High aldehyde dehydrogenase 1 (ALDH1) activity was recently shown to identify CSC-like cells in HNSCC (12). ALDH1-positivity also correlates with the number of cells undergoing epithelial-mesenchymal transition (EMT), a process that is considered a key prerogative for the formation of metastases $(13,14)$.

Since it is hypothesized that CSCs play a significant role in tumor progression, their frequency in primary tumors may also correlate with the extent of invasion and metastasis. Tumor cells undergoing EMT and its reverse process, mesenchymal- 
to-epithelial transition, are closely related to cells with CSC phenotype (13). We previously reported that $\mathrm{ALDH1} 1^{+}$putative CSCs expanded from HNSCC cell lines exhibited traits including self-renewal, quiescence, and increased expression of the stemness related genes Oct $3 / 4$, SOX 2 and NANOG. These cells also possess a higher invading capacity and upregulated EMT-marker expression, such as Snail1 and Twist, as well as a significantly increased expression of mesenchymal markers such as $\alpha$-smooth muscle actin and vimentin (14). Yang et al (15) found as an essential mechanism for EMT that Rac1 activation mediated Twist1-induced cancer cell migration. Twist1-induced activated tumor cells had a motile stem-like cancer cell phenotype that correlated with tumor invasiveness and unfavorable outcome in head and neck cancer patients. Although there is accumulating experimental evidence for the biological role of the CSC model, clinical confirmation of the role of CSCs in malignant progression and metastasis is limited.

One of the main etiologies of HNSCC is a persisting infection with oncogenic HR-HPV defining a distinct subgroup of the disease. HPV association has been detected in $20-30 \%$ of tumors located in all head and neck anatomic subsites and in approximately $50 \%$ of OSCCs, which were therefore chosen for this study (1). Currently, 15 confirmed HR-HPV-types are confirmed carcinogens. As in cervical cancer, HPV type 16 seems to play the major role in the etiology of HR-HPVassociated HNSCC. In general, patients affected by $\mathrm{HPV}^{+}$or HPV HNSCC differ in incidence, age, genetic background and prognosis $(5,16,17)$. Therefore, it is of interest to investigate comparatively the biological and clinical differences of CSCs of HR-HPV ${ }^{+}$and HR-HPV- OSCC as well as differences between primary tumors and their metastases.

We designed this study in paired samples of primary OSCC and their respective lymph node metastases with the aim to evaluate the relevance of CSC content in various stages of HPV-related and unrelated OSCC. Understanding the associations and relevance between HPV status and CSC content, in the progression of OSCC could support a rationale for the development of specific therapies for the distinct subgroups of HNSCC and also for therapies targeting CSC directly.

\section{Materials and methods}

Patient characteristics. This study was approved by the Internal Review Board of the University of Cologne, Germany. Study participants were between 38 and 79 years of age (median 57.8 years; male to female ratio $2.6: 1$ ) and only patients with no prior history of malignancies were included. We evaluated 40 paired samples of primary OSCC and corresponding lymph node metastases. Data on the histological stage of tumor, differentiation and the TNM classification were retrieved from the pathology database and patient charts.

HPV detection and typing. HPV DNA was amplified using a highly sensitive, group-specific nested PCR with degenerated primers A5/A10 and A6/A8 as previously described $(18,19)$. Briefly, direct sequence analysis of purified PCR products (QIAquick PCR Purification kit, Qiagen, Hilden, Germany) was carried out with an ABI Prism 377 DNA sequencer using the Taq FS BigDye Terminator cycle sequencing method (PE Applied Biosystems, Weiterstadt, Germany). Additionally,
A6/A8 PCR products (270 base pairs) were cloned into the vector pCR-Blunt II-Topo using the Zero Blunt Topo PCR Cloning kit (Invitrogen, Leek, The Netherlands). Plasmid DNA harboring an EcoRI insert of the expected size was sequenced as mentioned above. For HPV typing, obtained sequence information was compared with an HPV database (20).

Immunohistochemistry. Tissue specimens were fixed in $4 \%$ buffered formaldehyde and embedded in paraffin. Sections ( $4 \mu \mathrm{m}$ thick) were mounted on Superfrost Plus glass slides (Microm, Walldorf, Germany), deparaffinized and rehydrated in a series of graded ethanol. Immunohistochemical staining was performed using the two-step IHC detection reagent following the manufacturer's instructions (EnVision System-HRP Mouse, Dako, Hamburg, Germany). Briefly, after microwave treatment (twice for $7 \mathrm{~min}$ at $600 \mathrm{~W}$ in $10 \mathrm{mM}$ citrate buffer, $\mathrm{pH}$ 6.0) for antigen retrieval, endogenous peroxidase activity was blocked by immersing slides in ChemMate Peroxidase-Blocking Solution (Dako) $10 \mathrm{~min}$ at room temperature. Slides were incubated with mouse monoclonal antibody specific for p16 (1:100 dilution, clone DCS-50; Neomarkers, Fremont, CA, USA) or mouse monoclonal antibody specific for ALDH1 A1 (1:100 dilution, clone 44; BD Biosciences, San Jose, CA, USA) for $2 \mathrm{~h}$, followed by addition of HRP-labeled rabbit anti-mouse secondary antibody. Immunoreactive proteins were visualized with 3,3-diaminobenzidine and counterstained with Mayer's haematoxylin. Then, the sections were dehydrated and mounted. Positive and negative controls were included in each run for quality control of the immunoreactivity. Normal tonsils served as positive controls and a mouse isotype control (Dako) was used to replace the primary antibody as negative controls.

Evaluation of staining. Three independent experienced observers, who were blinded to the patient clinical information, performed semiquantitative evaluation of the slides. Discrepancies were resolved by a consensus meeting using a multiheaded microscope. Areas of carcinoma tissue within the samples and the p16 and ALDH1 expression pattern were evaluated by comparing the intensity and cellular localization of immunoreactivity with positive and negative controls. In general, $>1,000$ cells in five randomly selected fields of tumor tissue were analyzed for each section at a magnification of x400 to determine percentage labeling indices. The immunoreactivity of positively stained cells was graded into four categories to enable statistical analysis: grade $0,<5 \%$ positive cells; grade 1,5-25\%; grade 2, 26-50\%; and grade 3,>50\% In tumors showing heterogeneous expression, the grade was judged according to the predominant pattern.

Data analysis. Statistical analysis was performed using the Stata 9.0-software (StataCorp LP, College Station, TX, USA). Categorical variables were described by percentages and frequencies, and numerical variables were represented as means $\pm \mathrm{SD}$. Qualitative data were compared using the Chi-square or the Fisher's exact test, as appropriate. For continuous data, between-group comparisons were performed by either the Mann-Whitney or the Student's t-test, depending on the normality of each variable. All statistical comparisons were 2 -sided. A P-value of $<0.05$ was considered to indicate statistically significant differences. 
Table I. HPV-DNA detection and expression of HPV, p16 and ALDH1 in primary tumors and metastases.

\begin{tabular}{|c|c|c|c|c|c|c|c|c|c|}
\hline & \multicolumn{3}{|c|}{ HPV-DNA detection } & \multicolumn{3}{|c|}{ p16 expression } & \multicolumn{3}{|c|}{ ALDH1 expression } \\
\hline &,$- \mathrm{n}(\%)$ &,$+ \mathrm{n}(\%)$ & P-value &,$- \mathrm{n}(\%)$ &,$+ \mathrm{n}(\%)$ & P-value &,$- \mathrm{n}(\%)$ &,$+ \mathrm{n}(\%)$ & P-value \\
\hline Total $(\mathrm{N}=80)$ & $\mathrm{N}=48$ & $\mathrm{~N}=32$ & & $\mathrm{~N}=50$ & $\mathrm{~N}=30$ & & $\mathrm{~N}=9$ & $\mathrm{~N}=71$ & \\
\hline \multicolumn{10}{|l|}{ Origin } \\
\hline Primary $(n=40)$ & $20(50)$ & $20(50)$ & 0.068 & $23(58)$ & $17(42)$ & 0.356 & $5(13)$ & $35(87)$ & 0.723 \\
\hline Metastasis $(n=40)$ & $28(70)$ & $12(30)$ & & $27(68)$ & $13(32)$ & & $4(10)$ & $36(90)$ & \\
\hline \multicolumn{10}{|l|}{ HPV-DNA detection } \\
\hline $\mathrm{HPV}^{+}(\mathrm{n}=32)$ & & & & $5(16)$ & $27(84)$ & $<0.001$ & $3(9)$ & $29(91)$ & 0.665 \\
\hline $\operatorname{HPV}^{-}(\mathrm{n}=48)$ & & & & $45(94)$ & $3(6)$ & & $6(12)$ & $42(88)$ & \\
\hline \multicolumn{10}{|l|}{ p16 expression } \\
\hline $\mathrm{p} 16^{+}(\mathrm{n}=30)$ & & & & & & & $2(7)$ & $28(93)$ & 0.315 \\
\hline $\mathrm{p} 16^{-}(\mathrm{n}=50)$ & & & & & & & $7(14)$ & $43(86)$ & \\
\hline Primary $(\mathrm{N}=40)$ & $\mathrm{N}=20$ & $\mathrm{~N}=20$ & & $\mathrm{~N}=23$ & $\mathrm{~N}=17$ & & $\mathrm{~N}=5$ & $\mathrm{~N}=35$ & \\
\hline Age (years) & $55.7 \pm 9.49$ & $59.95 \pm 10.46$ & 0.1863 & $56.48 \pm 9.05$ & $59.65 \pm 11.37$ & 0.333 & $58.4 \pm 5.08$ & $57.74 \pm 10.66$ & 0.894 \\
\hline \multicolumn{10}{|l|}{ Gender } \\
\hline Female $(n=11)$ & $6(55)$ & $5(45)$ & 1.000 & $7(64)$ & $4(36)$ & 0.726 & $2(18)$ & $9(82)$ & 0.603 \\
\hline Male $(n=29)$ & $14(48)$ & $15(52)$ & & $16(53)$ & $13(47)$ & & $3(10)$ & $26(90)$ & \\
\hline \multicolumn{10}{|l|}{ Primary site } \\
\hline Tongue $(\mathrm{n}=7)$ & $5(71)$ & $2(29)$ & 0.407 & $4(57)$ & $3(43)$ & 1.000 & 0 & $7(100)$ & 0.565 \\
\hline Tonsil $(\mathrm{n}=33)$ & $15(45)$ & $18(55)$ & & $19(58)$ & $14(42)$ & & $5(15)$ & $28(85)$ & \\
\hline \multicolumn{10}{|l|}{ Tumor grade } \\
\hline $\mathrm{G} 1(\mathrm{n}=1)$ & 0 & $1(100)$ & 0.591 & 0 & $1(100)$ & 0.438 & $1(100)$ & 0 & 0.009 \\
\hline G1-2, G2 (n=19) & $10(53)$ & $9(47)$ & & $12(63)$ & $7(37)$ & & $2(11)$ & $17(89)$ & \\
\hline $\mathrm{G} 2-3, \mathrm{G} 3(\mathrm{n}=20)$ & $10(50)$ & $10(50)$ & & $11(55)$ & $9(45)$ & & $2(7)$ & $28(93)$ & \\
\hline \multicolumn{10}{|l|}{ Tumor stage } \\
\hline $\mathrm{T} 1(\mathrm{n}=11)$ & $4(36)$ & $7(64)$ & 0.505 & $5(45)$ & $6(55)$ & 0.637 & $2(18)$ & $9(82)$ & 0.270 \\
\hline $\mathrm{T} 2(\mathrm{n}=21)$ & $11(52)$ & $10(48)$ & & $13(62)$ & $8(38)$ & & $1(5)$ & $20(95)$ & \\
\hline $\mathrm{T} 3(\mathrm{n}=8)$ & $5(63)$ & $3(37)$ & & $5(63)$ & $3(37)$ & & $2(25)$ & $6(75)$ & \\
\hline \multicolumn{10}{|l|}{ HPV-DNA detection } \\
\hline $\mathrm{HPV}^{+}(\mathrm{n}=20)$ & & & & $4(20)$ & $16(80)$ & $<0.001$ & $3(15)$ & $17(85)$ & 1.000 \\
\hline $\mathrm{HPV}^{-}(\mathrm{n}=20)$ & & & & $19(95)$ & $1(5)$ & & $2(10)$ & $18(90)$ & \\
\hline \multicolumn{10}{|l|}{ p16 expression } \\
\hline $\mathrm{p} 16^{+}(\mathrm{n}=17)$ & & & & & & & $2(12)$ & $15(88)$ & 1.000 \\
\hline $\mathrm{p} 16^{-}(\mathrm{n}=23)$ & & & & & & & $3(13)$ & $20(87)$ & \\
\hline Metastasis $(\mathrm{N}=40)$ & $\mathrm{N}=28$ & $\mathrm{~N}=12$ & & $\mathrm{~N}=27$ & $\mathrm{~N}=13$ & & $\mathrm{~N}=4$ & $\mathrm{~N}=36$ & \\
\hline \multicolumn{10}{|l|}{$\mathrm{N}$-Stage } \\
\hline $\mathrm{N} 1(\mathrm{n}=15)$ & $12(80)$ & $3(20)$ & 0.477 & $11(73)$ & $4(27)$ & 0.730 & $4(27)$ & $11(73)$ & 0.015 \\
\hline $\mathrm{N} 2, \mathrm{~N} 3(\mathrm{n}=25)$ & $16(64)$ & $9(36)$ & & $16(64)$ & $9(36)$ & & 0 & $25(100)$ & \\
\hline \multicolumn{10}{|l|}{ HPV-DNA detection } \\
\hline $\mathrm{HPV}^{+}(\mathrm{n}=12)$ & & & & $1(8)$ & $11(92)$ & $<0.001$ & 0 & $12(100)$ & 0.297 \\
\hline $\mathrm{HPV}^{-}(\mathrm{n}=28)$ & & & & $26(93)$ & $2(7)$ & & $4(14)$ & $24(86)$ & \\
\hline \multicolumn{10}{|l|}{ p16 expression } \\
\hline $\mathrm{p} 16^{+}(\mathrm{n}=13)$ & & & & & & & 0 & $13(100)$ & 0.284 \\
\hline $\mathrm{p} 16^{-}(\mathrm{n}=27)$ & & & & & & & $4(15)$ & $23(85)$ & \\
\hline
\end{tabular}

\section{Results}

In $17.5 \%$ of the cases, the OSCC was located in the tongue, whereas in $82.5 \%$ it was located in the tonsil. The pathological tumor stage according to the TNM classification was as follows: pT1, $11(27.5 \%)$; pT2, $21(52.5 \%)$; pT3, 8 (20\%); $\mathrm{pN} 1,15$ (37.5\%); pN2+pN3, 25 (62.5\%). As tonsil tumors tend to be non-keratinizing or basaloid, grading is sometimes ambiguous. Therefore, 6 cases $(22 \%)$ were classified as grade 1-2 or 2-3 (Table I). 
Table II. Correlation between ALDH1 grade and clinicopathological characteristics.

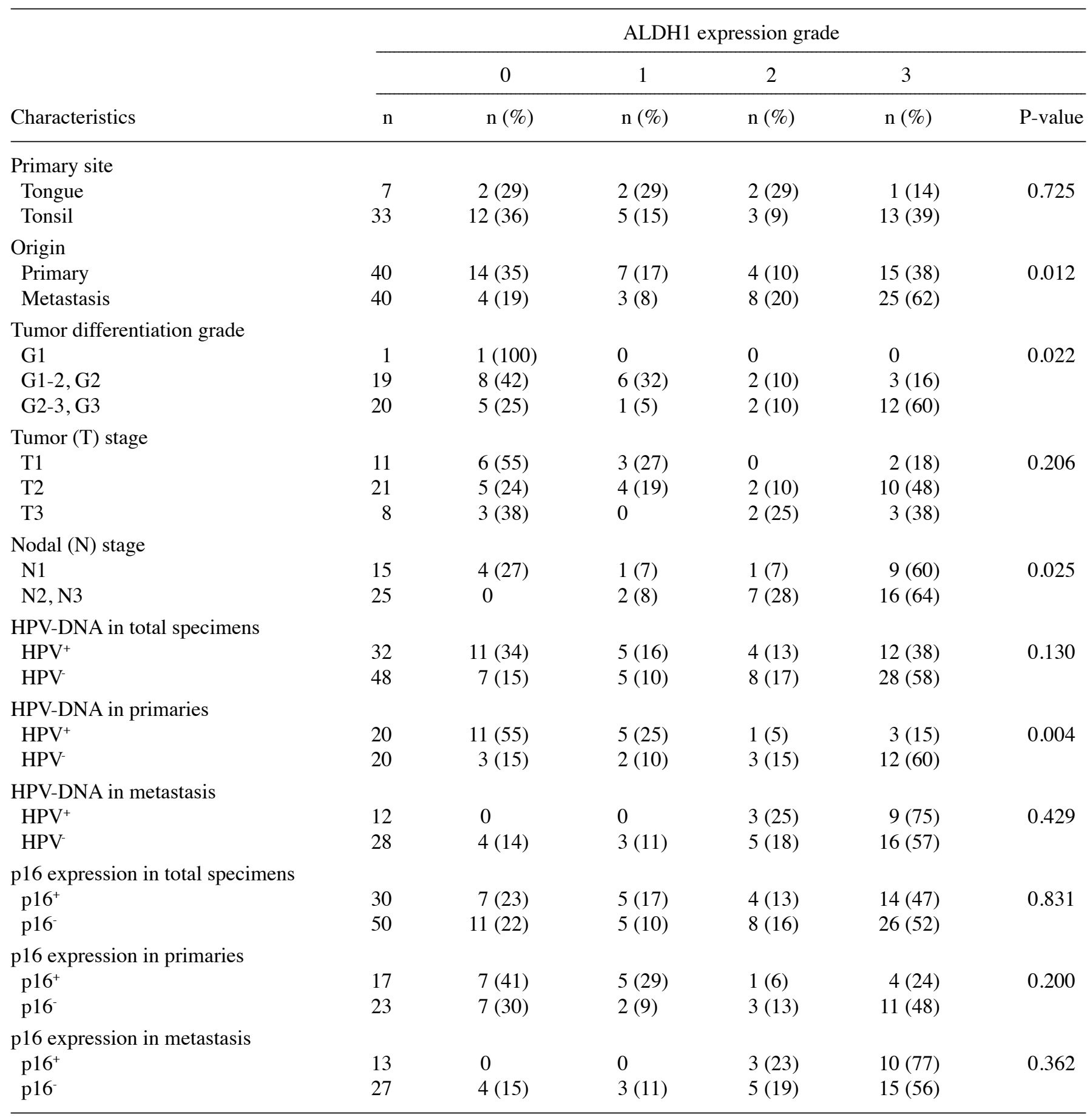

The correlation between positive HR-HPV status and p16 expression in total specimens $(\mathrm{P}<0.001)$, primary tumors $(\mathrm{P}<0.001)$ and metastases was highly significant $(\mathrm{P}<0.001)$ (Table I). A total of $80 \%$ of HR-HPV-DNA positive primary tumors co-expressed p16 and 95\% of HPV-DNA negative tumors were p16-negative. Furthermore, 92\% of HR-HPVpositive metastases were p16-positive and 93\% of HPV-DNA negative metastases were p16-negative. Positive p16-status has a specificity of $90 \%$ for HR-HPV-DNA positivity in this study. All 20 HPV-DNA negative primary tumors had negative HPV-specific PCR findings for their metastases, and all 23 p16-negative primary tumors had p16-negative metastases. Eight of 20 HR-HPV-DNA positive primary tumors had metastases where no HPV-DNA was detectable. In 4 out of 17 p16-positive primary tumors, the corresponding metastasis was p16-negative. No significant association was observed between HPV-DNA status or p16 expression and clinicopathological parameters.

Expression of ALDH1 was detected in $87.5 \%$ of primary tumors and in $90 \%$ of the metastases. ALDH1 positivity was significantly correlated with lower tumor differentiation $(\mathrm{P}=0.009)$ and higher $\mathrm{N}$ classification $(\mathrm{P}=0.015)$ (Table $\mathrm{I})$. Subsequently, samples were divided into groups with different ALDH1 expression grades (0-3). Higher expression grades of ALDH1 were also more frequent in primary tumors with lower 
Table III. Correlation between HPV-DNA detection with p16 and ALDH1 expression.

\begin{tabular}{|c|c|c|c|c|c|c|c|c|c|}
\hline \multirow[b]{3}{*}{ Characteristics } & \multirow[b]{3}{*}{$\mathrm{n}$} & \multirow[b]{3}{*}{$\mathrm{ALDH}^{+}$} & \multirow[b]{3}{*}{ ALDH1 $^{-}$} & \multirow[b]{3}{*}{ P-value } & \multicolumn{5}{|c|}{ ALDH1 expression grade } \\
\hline & & & & & 0 & 1 & 2 & 3 & \\
\hline & & & & & $\mathrm{n}(\%)$ & $\mathrm{n}(\%)$ & $\mathrm{n}(\%)$ & $\mathrm{n}(\%)$ & P-value \\
\hline \multicolumn{10}{|l|}{ Primary tumor } \\
\hline 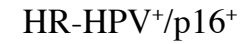 & 16 & $14(88)$ & $2(12)$ & 1.000 & 7 (44) & $5(31)$ & $1(6)$ & $3(19)$ & 0.038 \\
\hline $\mathrm{HPV}^{-/ p} 16^{-}$ & 19 & $17(89)$ & $2(11)$ & & $6(32)$ & $2(11)$ & $3(16)$ & $11(58)$ & \\
\hline \multicolumn{10}{|l|}{ Metastasis } \\
\hline $\mathrm{HR}-\mathrm{HPV}^{+} / \mathrm{p} 16^{+}$ & 11 & $11(100)$ & 0 & 0.296 & 0 & 0 & $3(27)$ & $8(73)$ & 0.441 \\
\hline $\mathrm{HPV} / \mathrm{p} 16^{-}$ & 26 & $22(85)$ & $4(15)$ & & $4(15)$ & $3(11)$ & $5(19)$ & $14(54)$ & \\
\hline
\end{tabular}

A

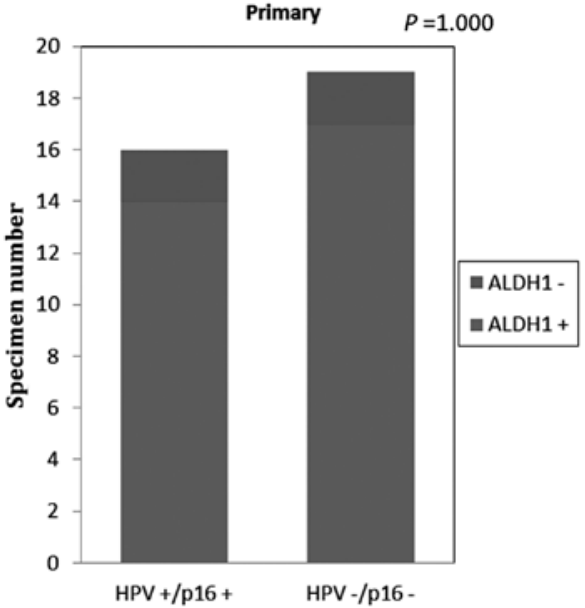

C

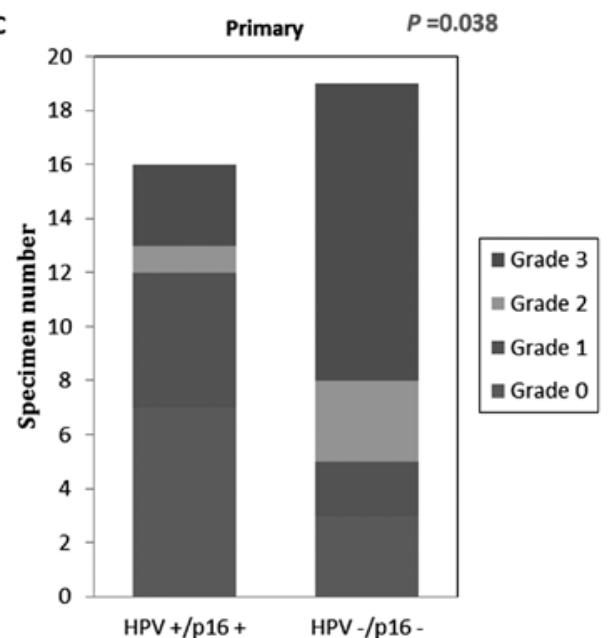

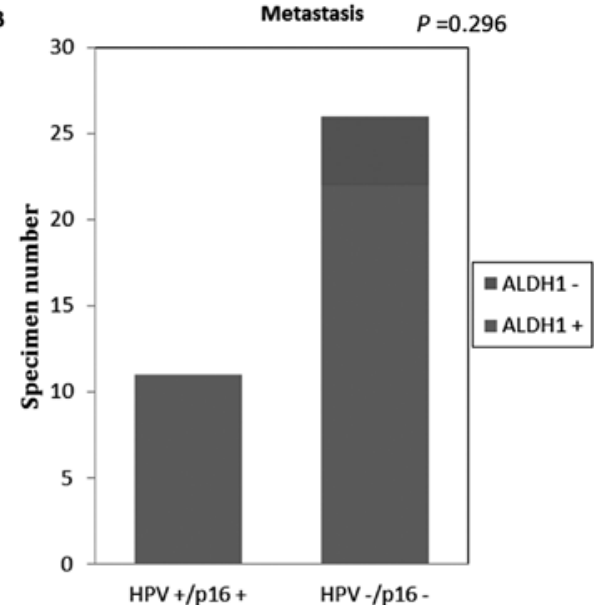

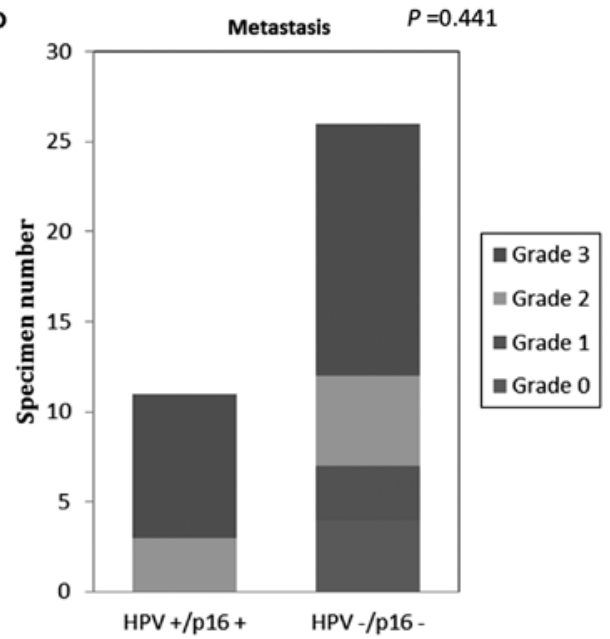

Figure 1. Correlation between HPV-DNA detection with p16 and aldehyde dehydrogenase 1 (ALDH1) expression. Distribution of ALDH1 positivity between HR-HPV-related $\left(\mathrm{HPV}^{+} / \mathrm{p}^{+} 6^{+}\right)$and HPV-unrelated (HPV $/$p16 $)$tumors in (A) primary oropharyngeal squamous cell carcinoma (OSCC) or (B) nodal metas-

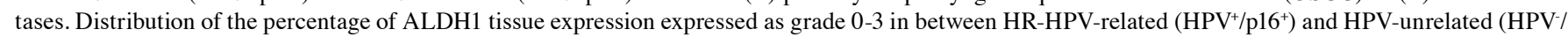
p16) tumors in (C) primary OSCC or (D) nodal metastases.

tumor differentiation $(\mathrm{P}=0.022)$ and higher $\mathrm{N}$ classification, $(\mathrm{P}=0.025)$ (Table II). Next, we analyzed the HPV-status, p16, and ALDH1 positivity in metastases, however, no significant correlation was found. By contrast, ALDH1 expression grades were significantly elevated in metastases vs. primary tumors $(\mathrm{P}=0.012)$ (Table III).
Comparing HPV-negative and -positive tumors, there was no correlation between ALDH1 expression and HPV status in total specimens $(\mathrm{P}=0.665)$, primary tumors $(\mathrm{P}=1)$ and metastases $(P=0.297)$ (Table I). Significant correlations, however, were found between higher ALDH1 expression grades and negative HPV status for primary tumors $(\mathrm{P}=0.004)$, but not 

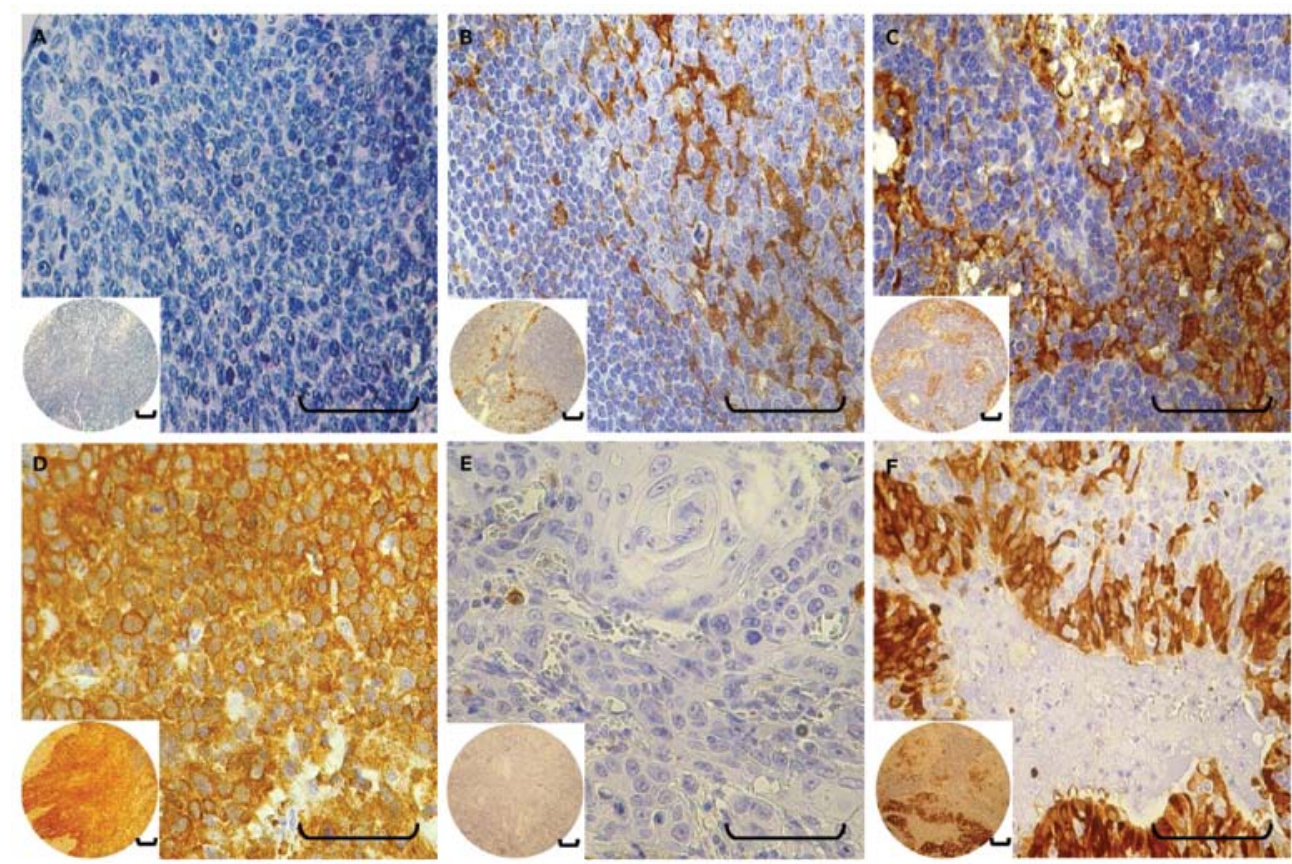

Figure 2. Representative examples of aldehyde dehydrogenase 1 (ALDH1)-specific immunostaining in oropharyngeal squamous cell carcinoma (OSCC) (A) with grade 0; (B) with grade 1; (C) with grade 2; and (D) with grade 3. (E) Distribution of ALDH1 expression in primary tumor tissue. (F) Representative ALDH1 expression in corresponding metastasis. Scale bar, $100 \mu \mathrm{m}$.

for metastases $(\mathrm{P}=0.429)$ (Table II). There was no correlation between ALDH1 expression and p16 expression for primary tumors $(\mathrm{P}=1)$ and metastases $(\mathrm{P}=0.284)$ (Table I). There was no significant correlation between the ALDH1 expression grade and p16 expression between the primary tumors $(\mathrm{P}=0.2)$ and metastases $(\mathrm{P}=0.362)$ (Table II).

Subgroups of HR-HPV-DNA ${ }^{+} / \mathrm{p}^{+} 6^{+}$and HPV-DNA $/ \mathrm{p} 16$ tumors were correlated with ALDH1 expression. There were no correlations between these two groups concerning ALDH1positive and -negative expression in primary tumors or metastases. However, when comparing these subgroups concerning ALDH1 expression grades, we found that $\mathrm{HPV}-\mathrm{DNA}^{+} / \mathrm{p} 6^{+}$primary tumors exhibited significantly lower ALDH1 expression grades, whereas HPV-DNA-/p16- primary tumors presented with higher ALDH1 grades $(\mathrm{P}=0.038)$ (Fig. 1). There were no similar findings for metastases $(\mathrm{P}=0.441)$. Representative examples are shown in Fig. 2.

\section{Discussion}

In elucidating $\mathrm{HNSCC}$ tumorigenesis, CSC research is currently a promising field that could lead to a better understanding of the formation of recurrence, metastasis, and resistance to radiotherapy and chemotherapy. Prince et al (11) were among the first to demonstrate that the $\mathrm{CD} 44^{+}$cell population in HNSCC possesses properties of cancer stem cells. Later, Chen et al (12) showed that $\mathrm{ALDH}^{+}$cells derived from HNSCC were tumorigenic and displayed resistance towards radiotherapy. In another study, the authors found that silencing of Bmi-1, a transcriptional repressor essential for maintaining the self-renewal abilities of adult stem cells and CSCs, significantly increased the sensitivity of $\mathrm{ALDH}^{+} \mathrm{HNSCC}$ cells to chemo-radiation and the degree of chemo-radiation-mediated apoptosis (21). Therefore, measuring ALDH1 expression is promising as a marker for therapeutic success and prognosis.

In the current study, we specifically focused on the clinical and histopathological characteristics of OSCC in relation to ALDH1 expression. We investigated the presence of ALDH1 ${ }^{+}$ CSCs in primary OSCC as a subgroup of HNSCC that is in approximately half of the cases HR-HPV-positive, and in corresponding lymph node metastases from clinical paraffinembedded specimens. We demonstrated that higher ALDH1 grades are significantly associated with a lower grade of tumor differentiation and a higher nodal classification of the OSCC. This indicates that the frequency of CSCs is related to poorly differentiated OSCC and the occurrence of nodal metastasis and that the frequency of CSCs is related to the progression of OSCC and possibly plays a role in this process. In support of these findings, numerous recent studies of carcinomas of different origin also presented traits of high-grade malignancy that could be specifically traced to the presence of CSCs, indicating that CSCs may be the critical drivers of tumor progression $(22,23)$.

Moreover, CSCs have been defined by their key trait, the ability to seed new tumors and metastases (13). In our previous study in HNSCC cell lines, the invasiveness of tumor cells was explored and correlated with the CSC phenotype (14). CSCs reside in close proximity to blood vessels and can give rise to tumor endothelium (24). It was reported that endothelialderived factors inhibit anoikis of $\mathrm{ALDH}^{+} \mathrm{CD} 44^{+}$head and neck cancer stem cells (25). EMT is regarded a necessary process that empowers CSCs to disseminate from primary tumors and to seed metastases. In order to understand the role of $\mathrm{ALDH}^{+} \mathrm{CSC}$ in the progression of OSCC, we analyzed their frequency and distribution in primary tumors and their metastases. Only few previous studies have thus far compara- 
tively studied CSCs in primary tumors with their associated metastases. It was reported that increased ALDH1 expression in patients with breast cancer lymph node metastases serves as a prognostic factor of poor clinical outcome (26).

We have demonstrated the expression of ALDH1 in pairs of primary OSCC and corresponding lymph node metastases. In the total case collection, metastasis did not display a significantly increased number of $\mathrm{ALDH} 1^{+}$cells when compared to the primary tumor. However, if the percentage of $\mathrm{ALDH} 1^{+}$ cells in the materials were classified into grades $0-3$, significant differences between primary tumor and corresponding lymph node metastases became apparent. Lower ALDH1 grades in the primary were seen in $\mathrm{HPV}^{+}$tumors and higher grades in $\mathrm{HPV}^{-}$primaries. By contrast, this difference was not observed in metastases. In general a higher grade of ALDH1 expression was more frequently found in metastases and also correlated with a higher nodal metastasis status of the patient. The observation that the number of CSCs was increased in metastases may reflect the fact that successful seeding of metastases requires a more motile cellular phenotype that is supported by the ability of CSCs to undergo EMT. It remains to be explored if the increase of CSC numbers manifests transiently e.g. in the initiation period during the metastatic colonization and decreases later or displays a stable state. In accordance with our findings, Malanchi et al (27) found that in a breast cancer model, the relative size of the CSC population increased during the early period of metastatic colonization in the secondary target organ, i.e. the lung. In our study, metastases presented higher grades of ALDH1 expression than the corresponding primary tumor. This evidence could also be indicative of the active function of CSCs in the process of metastasis formation.

In the present study, we showed, for the first time, a significant association between ALDH1 ${ }^{+}$OSCC and their nodal metastases. Taken together, the results of our study of EMT capacity and ALDH association of HNSCC cell lines and the current data from clinical paraffin-embedded specimens, $\mathrm{ALDH} 1^{+} \mathrm{CSCs}$ are highly invasive and have metastatic ability. Recently, $\mathrm{Xu}$ et al (28) investigated the ALDH1 ${ }^{+} \mathrm{CSC}$ content of primary metastatic or non-metastatic HNSCC. ALDH1 expression in primary tumors correlated with lymph node metastasis. Patients with low ALDH1 expression levels in primary tumors had a better 5-year survival rate than those with high ALDH1 expression levels.

With regard to the etiology of OSCC, the HPV status was also explored in the present study. In the past, much attention has focused on the possible role of HPV infection in the pathogenesis of OSCC. HPV and protein p16 ${ }^{\mathrm{INKa}}$ (p16) are among the most commonly reported OSCC markers. In agreement with previous studies, our findings demonstrate that HPV and p16 are highly correlated in both primary OSCC and metastases $(5,29)$. Numerous studies reported that $\mathrm{HR}_{-} \mathrm{HPV}^{+} \mathrm{OSCC}$ patients showed better survival than those with $\mathrm{HPV}^{-}$OSCC, although $\mathrm{HPV}^{+}$tumors are less differentiated than $\mathrm{HPV}^{-}$tumors and present more frequently with nodal metastases $(30,31)$. These counterintuitive findings suggest HR-HPV associated tumors have distinct biological features. We observed that there was a significant correlation between a higher ALDH1 expression grade in the tissue and negative HPV status for primary tumors, but not for metastases. By subgroup analyses, we found $\mathrm{HR}-\mathrm{HPV}-\mathrm{DNA}^{+} / \mathrm{p} 16^{+}$primary tumors exhibited lower ALDH1 expression grades representing a lower number of CSCs, while HPV-DNA\%/p16- primary tumors had higher ALDH1 grades representing a higher CSC frequency. Our data support the existence of distinct tumor cell characteristics defined by the HPV status. This difference is reflected by different ALDH1 grading in the primary tumors.

Since $\mathrm{ALDH}^{+} \mathrm{CSC}$ might play an important role in the progression of malignancies, our findings may help to explain the better survival of $\mathrm{HPV}^{+}$OSCC patients. It is evident that only enumerating the frequency of CSC populations in HR-HPV-related OSCC (low in primary and high in metastases) does not yield a satisfying answer. The function of HPV in CSCs as well as the genetic and epigenetic changes in OSCC remain to be elucidated in future studies.

In conclusion, in this initial study we characterized the patterns of ALDH1, p16 expression and HPV-DNA status in primary OSCC and their lymph node metastases. Our data suggest that enumeration alone does not suffice, and that studying phenotypical and functional states of CSCs is critical in understanding their specific abilities that determine differences in the prognosis of HPV-related and unrelated OSCC. These insights may support therapeutic design and decisions in the future.

\section{Acknowledgements}

The authors thank Ms. Erika Berg for her technical assistance. Dr Xu Qian received a stipend from ZAST-organization dedicated to support scientific exchange between China and Germany.

\section{References}

1. Leemans CR, Braakhuis BJ and Brakenhoff RH: The molecular biology of head and neck cancer. Nat Rev Cancer 11: 9-22, 2011.

2. Chaturvedi AK, Engels EA, Pfeiffer RM, et al: Human papillomavirus and rising oropharyngeal cancer incidence in the United States. J Clin Oncol 29: 4294-4301, 2011.

3. Licitra L, Zigon G, Gatta G, Sanchez MJ and Berrino F; EUROCARE Working Group: Human papillomavirus in HNSCC: a European epidemiologic perspective. Hematol Oncol Clin North Am 22: 1143-1153, vii-viii, 2008.

4. Nasman A, Attner P, Hammarstedt L, et al: Incidence of human papillomavirus (HPV) positive tonsillar carcinoma in Stockholm, Sweden: an epidemic of viral-induced carcinoma? Int J Cancer 125: 362-366, 2009.

5. Ang KK, Harris J, Wheeler R, et al: Human papillomavirus and survival of patients with oropharyngeal cancer. N Engl J Med 363: 24-35, 2010

6. Gillison ML, D'Souza G, Westra W, et al: Distinct risk factor profiles for human papillomavirus type 16-positive and human papillomavirus type 16-negative head and neck cancers. J Natl Cancer Inst 100: 407-420, 2008.

7. Koukourakis MI, Giatromanolaki A, Tsakmaki V, Danielidis V and Sivridis E: Cancer stem cell phenotype relates to radiochemotherapy outcome in locally advanced squamous cell head-neck cancer. Br J Cancer 106: 846-853, 2012.

8. Nguyen LV, Vanner R, Dirks P and Eaves CJ: Cancer stem cells: an evolving concept. Nat Rev Cancer 12: 133-143, 2012.

9. Singh A and Settleman J: EMT, cancer stem cells and drug resistance: an emerging axis of evil in the war on cancer. Oncogene 29: 4741-4751, 2010.

10. Albers AE, Chen C, Koberle B, et al: Stem cells in squamous head and neck cancer. Crit Rev Oncol Hematol 81: 224-240, 2012.

11. Prince ME, Sivanandan R, Kaczorowski A, et al: Identification of a subpopulation of cells with cancer stem cell properties in head and neck squamous cell carcinoma. Proc Natl Acad Sci USA 104: 973-978, 2007. 
12. Chen YC, Chen YW, Hsu HS, et al: Aldehyde dehydrogenase 1 is a putative marker for cancer stem cells in head and neck squamous cancer. Biochem Biophys Res Commun 385: 307-313, 2009.

13. Chaffer CL and Weinberg RA: A perspective on cancer cell metastasis. Science 331: 1559-1564, 2011.

14. Chen C, Wei Y, Hummel M, et al: Evidence for epithelialmesenchymal transition in cancer stem cells of head and neck squamous cell carcinoma. PLoS One 6: e16466, 2011.

15. Yang WH, Lan HY, Huang $\mathrm{CH}$, et al: RACl activation mediates Twist1-induced cancer cell migration. Nat Cell Biol 14: 366-374, 2012.

16. Gillison ML, Koch WM, Capone RB, et al: Evidence for a causal association between human papillomavirus and a subset of head and neck cancers. J Natl Cancer Inst 92: 709-720, 2000.

17. Klussmann JP, Mooren JJ, Lehnen M, et al: Genetic signatures of HPV-related and unrelated oropharyngeal carcinoma and their prognostic implications. Clin Cancer Res 15: 1779-1786, 2009.

18. Klussmann JP, Weissenborn SJ, Wieland U, et al: Prevalence, distribution, and viral load of human papillomavirus 16 DNA in tonsillar carcinomas. Cancer 92: 2875-2884, 2001.

19. Wieland U, Ritzkowsky A, Stoltidis M, et al: Papillomavirus DNA in basal cell carcinomas of immunocompetent patients: an accidental association? J Invest Dermatol 115: 124-128, 2000.

20. Myers G, Baker C, Münger K, Sverdrup F, McBride A and Bernard HU (ed): Human Papillomaviruses 1997. Los Alamos National Laboratory, Los Alamos, NM, 1997.

21. Chen YC, Chang CJ, Hsu HS, et al: Inhibition of tumorigenicity and enhancement of radiochemosensitivity in head and neck squamous cell cancer-derived ALDH1-positive cells by knockdown of Bmi-1. Oral Oncol 46: 158-165, 2010.
22. Charafe-Jauffret E, Ginestier C, Iovino F, et al: Breast cancer cell lines contain functional cancer stem cells with metastatic capacity and a distinct molecular signature. Cancer Res 69: 1302-1313, 2009.

23. Pang R, Law WL, Chu AC, et al: A subpopulation of CD26 cancer stem cells with metastatic capacity in human colorectal cancer. Cell Stem Cell 6: 603-615, 2010.

24. Wang R, Chadalavada K, Wilshire J, et al: Glioblastoma stem-like cells give rise to tumour endothelium. Nature 468: 829-833, 2010.

25. Campos MS, Neiva KG, Meyers KA, Krishnamurthy S and Nor JE: Endothelial derived factors inhibit anoikis of head and neck cancer stem cells. Oral Oncol 48: 26-32, 2012.

26. Nogami T, Shien T, Tanaka T, et al: Expression of ALDH1 in axillary lymph node metastases is a prognostic factor of poor clinical outcome in breast cancer patients with 1-3 lymph node metastases. Breast Cancer: Mar 10, 2012 (Epub ahead of print).

27. Malanchi I, Santamaria-Martinez A, Susanto E, et al: Interactions between cancer stem cells and their niche govern metastatic colonization. Nature 481: 85-89, 2012.

28. Xu J, Muller S, Nannapaneni S, et al: Comparison of quantum dot technology with conventional immunohistochemistry in examining aldehyde dehydrogenase 1A1 as a potential biomarker for lymph node metastasis of head and neck cancer. Eur J Cancer 8: 1682-1689, 2012.

29. D'Souza G, Kreimer AR, Viscidi R, et al: Case-control study of human papillomavirus and oropharyngeal cancer. N Engl J Med 356: 1944-1956, 2007.

30. Begum S and Westra WH: Basaloid squamous cell carcinoma of the head and neck is a mixed variant that can be further resolved by HPV status. Am J Surg Pathol 32: 1044-1050, 2008.

31. Mendelsohn AH, Lai CK, Shintaku IP, et al: Histopathologic findings of HPV and p16 positive HNSCC. Laryngoscope 120: 1788-1794, 2010 